\title{
The density variance - Mach number relation in the Taurus molecular cloud
}

\author{
C. M. Brunt
}

\begin{abstract}
Astrophysics Group, School of Physics, University of Exeter, Stocker Road, Exeter, EX4 4QL, UK
e-mail: brunt@astro.ex.ac.uk
\end{abstract}

Received 20 October 2009 / Accepted 2 February 2010

\begin{abstract}
Supersonic turbulence in molecular clouds is a key agent in generating density enhancements that may subsequently go on to form stars. The stronger the turbulence - the higher the Mach number - the more extreme the density fluctuations are expected to be. Numerical models predict an increase in density variance, $\sigma_{\rho / \rho_{0}}^{2}$, with $\mathrm{rms}$ Mach number, $M$ of the form: $\sigma_{\rho / \rho_{0}}^{2}=b^{2} M^{2}$, where $b$ is a numerically-estimated parameter, and this prediction forms the basis of a large number of analytic models of star formation. We provide an estimate of the parameter $b$ from ${ }^{13} \mathrm{CO} J=1-0$ spectral line imaging observations and extinction mapping of the Taurus molecular cloud, using a recently developed technique that needs information contained solely in the projected column density field to calculate $\sigma_{\rho / \rho_{0}}^{2}$. When this is combined with a measurement of the rms Mach number, $M$, we are able to estimate $b$. We find $b=0.48_{-0.11}^{+0.15}$, which is consistent with typical numerical estimates, and is characteristic of turbulent driving that includes a mixture of solenoidal and compressive modes. More conservatively, we constrain $b$ to lie in the range 0.3-0.8, depending on the influence of sub-resolution structure and the role of diffuse atomic material in the column density budget (accounting for sub-resolution variance results in higher values of $b$, while inclusion of more low column density material results in lower values of $b$; the value $b=0.48$ applies to material which is predominantly molecular, with no correction for sub-resolution variance). We also report a break in the Taurus column density power spectrum at a scale of $\sim 1 \mathrm{pc}$, and find that the break is associated with anisotropy in the power spectrum. The break is observed in both ${ }^{13} \mathrm{CO}$ and dust extinction power spectra, which, remarkably, are effectively identical despite detailed spatial differences between the ${ }^{13} \mathrm{CO}$ and dust extinction maps.
\end{abstract}

Key words. magnetohydrodynamics (MHD) - turbulence - techniques: spectroscopic - ISM: molecules - radio lines: ISM ISM: kinematics and dynamics

\section{Introduction}

Recent years have seen a proliferation of analytical models of star formation that provide prescriptions for star formation rates and initial mass functions based on physical properties of molecular clouds (Padoan \& Nordlund 2002; Krumholz \& McKee 2005; Elmegreen 2008; Hennebelle \& Chabrier 2008; Padoan \& Nordlund 2009; Hennebelle \& Chabrier 2009). While these models differ in their details, they are all fundamentally based on the same increasingly influential idea that has emerged from numerical models: that the density PDF is lognormal in form for isothermal gas (Vázquez-Semadeni 1994), with the normalized density variance increasing with the rms Mach number $\left(\sigma_{\rho / \rho_{0}}^{2}=b^{2} M^{2}\right.$ where $\rho$ is the density, $\rho_{0}$ is the mean density, $M$ is the $3 \mathrm{D}$ rms Mach number, and $b$ is a numerically determined parameter; Padoan et al. 1997b).

There is some uncertainty on the value of $b$. Padoan et al. (1997a,b) propose $b=0.5$ in $3 \mathrm{D}$, while Passot \& Vázquez-Semadeni (1998) found $b=1$ using 1D simulations. Federrath et al. (2008) suggested that $b=1 / 3$ for solenoidal (divergence-free) forcing and $b=1$ for compressive (curl-free) forcing in 3D. A value of $b=0.25$ was recently found by Kritsuk et al. (2007) in numerical simulations that employed a mixture of compressive and solenoidal forcing. Lemaster \& Stone (2008) found an non-linear relation between $\sigma_{\rho / \rho_{0}}^{2}$ and $M^{2}$, but it is very similar to a linear relation with $b=1 / 3$ over the range of Mach numbers they analyzed. They also found that magnetic fields appear to have only a weak effect on the $\sigma_{\rho / \rho_{0}}^{2}-M^{2}$ relation, and noted that the relation may be different in conditions of decaying turbulence.

Currently, observational information on the value of $b$, or the linearity of the $\sigma_{\rho / \rho_{0}}^{2}-M^{2}$ relation, is very sparse. A major obstacle is the inaccessibility of the 3D density field. Recently, Brunt et al. (2009, BFP) have developed and tested a method of calculating $\sigma_{\rho / \rho_{0}}^{2}$ from information contained solely in the projected column density field, which we apply to the Taurus molecular cloud in this paper. Using a technique similar to that of BFP, Padoan et al. (1997a) have previously estimated a value of $b=0.5\left(M=10, \sigma_{\rho / \rho_{0}}=5\right)$ for the IC 5146 molecular cloud. In sub-regions of the Perseus molecular cloud Goodman et al. (2009) found no obvious relation between Mach number and normalized column density variance, $\sigma_{N / N_{0}}^{2}$. It has been suggested by Federrath et al. (2008) that this could be due to differing levels of compressive forcing, but it may also be due to differing proportions of $\sigma_{\rho / \rho_{0}}^{2}$ projected into $\sigma_{N / N_{0}}^{2}$ (BFP). Alternatively, this may imply that there is no obvious relation between $\sigma_{\rho / \rho_{0}}^{2}$ and $M^{2}$.

The aim of this paper is to constrain the $\sigma_{\rho / \rho_{0}}^{2}-M^{2}$ relation observationally. In Sect. 2, we briefly describe the BFP technique, and in Sect. 3 we apply it to spectral line imaging observations (Narayanan et al. 2008; Goldsmith et al. 2008) and dust 
extinction mapping (Froebrich et al. 2007) of the Taurus molecular cloud to establish an observational estimate of $b$. Section 4 provides a summary.

\section{Measuring the 3D density variance}

We do not have access to the 3D density field, $\rho(x, y, z)$ in a molecular cloud to measure $\sigma_{\rho / \rho_{0}}^{2}$ directly, but must instead rely on information contained the projected $2 \mathrm{D}$ column density field, $N(x, y)$. One can use Parseval's Theorem to relate the observed variance in the normalized column density field, $\sigma_{N / N_{0}}^{2}$ to the sum of its power spectrum, $P_{N / N_{0}}\left(k_{x}, k_{y}\right)$. It can be shown that $P_{N / N_{0}}\left(k_{x}, k_{y}\right)$ is proportional to the $k_{z}=0$ cut through the $3 \mathrm{D}$ power spectrum, $P_{\rho / \rho_{0}}\left(k_{x}, k_{y}, k_{z}\right)$. Since $\sigma_{\rho / \rho_{0}}^{2}$ can in turn be related, again through Parseval's Theorem, to the sum of its power spectrum, $P_{\rho / \rho_{0}}$, it is possible, assuming an isotropic density field, to calculate the ratio, $R=\sigma_{N / N_{0}}^{2} / \sigma_{\rho / \rho_{0}}^{2}$ from measurements made on the column density field alone. It was shown by $\mathrm{BFP}$ that $R$ is given by:

$$
\frac{\left[\sum_{k_{x}=-\lambda / 2+1}^{\lambda / 2} \sum_{k_{y}=-\lambda / 2+1}^{\lambda / 2}\left\langle P_{N / N_{0}}\right\rangle(k) \tilde{B}^{2}(k)\right]-P_{N / N_{0}}(0) \tilde{B}^{2}(0)}{\left[\sum_{k_{x}=-\lambda / 2}^{\lambda / 2} \sum_{k_{y}=-\lambda / 2}^{\lambda / 2} \sum_{k_{z}=-\lambda / 2+1}^{\lambda / 2}\left\langle P_{N / N_{0}}\right\rangle(k) \tilde{B}^{2}(k)\right]-P_{N / N_{0}}(0) \tilde{B}^{2}(0)},
$$

where $\lambda$ is the number of pixels along each axis, $\left\langle P_{N / N_{0}}\right\rangle(k)$ is the azimuthally-averaged power spectrum of $N / N_{0}$, and $\widetilde{B}^{2}(k)$ is the square of the Fourier space representation of the telescope beam pattern. In Eq. (1), it should be noted that $\left\langle P_{N / N_{0}}\right\rangle(k)$ is the power spectrum of the column density field in the absence of beam-smoothing and instrumental noise.

An observational measurement of $\sigma_{N / N_{0}}^{2}$ and $R$ can then yield $\sigma_{\rho / \rho_{0}}^{2}$. To calculate the power spectrum in Eq. (1), zero-padding of the field may be necessary, to reduce edge discontinuities and to make the field square. If a field of size $\lambda_{x} \times \lambda_{y}$ is zero-padded to produce a square field of size $\lambda_{p x} \times \lambda_{p y}$ then $\sigma_{\rho / \rho_{0}}^{2}$ should be calculated via:

$\sigma_{\rho / \rho_{0}}^{2}=\frac{1}{\eta^{3}}\left(1+\left(\left(\sigma_{N / N_{0}}^{2}+1\right) \eta^{2}-1\right) / R_{p}\right)-1$

where $\eta=\left(\lambda_{p x} \lambda_{p y} / \lambda_{x} \lambda_{y}\right)^{\frac{1}{2}}$, and $R_{p}$ is the 2D-to-3D variance ratio calculated from the power spectrum of the padded field using Eq. (1). This assumes that the line-of-sight extent (in pixels) of the density field is $\lambda_{z}=\left(\lambda_{x} \lambda_{y}\right)^{\frac{1}{2}}$.

The BFP method assumes isotropy in the 3D density field, so Eq. (1) must be applied with some caution. Using magnetohydrodynamic turbulence simulations, BFP show that the assumption of isotropy is valid if the turbulence is super-Alfvénic $\left(M_{\mathrm{A}}>1\right)$ or, failing this, is strongly supersonic $(M \gtrsim 10)$. The BFP method can be used to derive $\sigma_{\rho / \rho_{0}}^{2}$ to around $10 \%$ accuracy if these criteria are met, while up to a factor of 2 uncertainty may be expected in the sub-Alfvénic, low sonic Mach number regime. It should also be noted that the variance calculated at finite resolution is necessarily a lower limit to the true variance. While this problem can only be addressed by higher resolution observations, estimates of the expected shortfall in the variance can be made from available information.

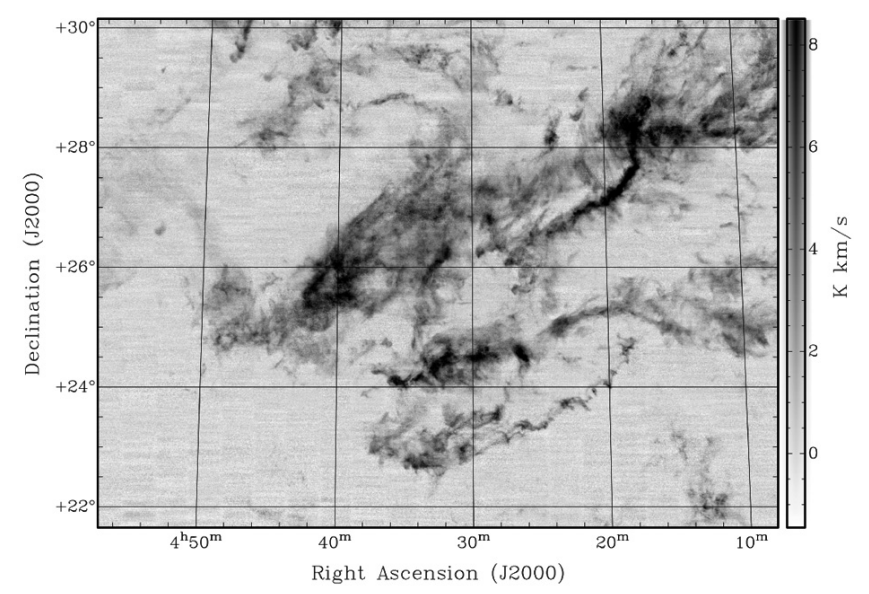

Fig. 1. Integrated intensity map of the ${ }^{13} \mathrm{CO} J=1-0$ line over the velocity range $[0,12] \mathrm{km} \mathrm{s}^{-1}$ in the Taurus molecular cloud.

\section{Application to the Taurus molecular cloud}

\subsection{Measurement of $b$ using ${ }^{13} \mathrm{CO}$ data only}

We now apply the BFP method to ${ }^{13} \mathrm{CO}(J=1-0)$ spectral line imaging observations of the Taurus molecular cloud. Figure 1 shows the ${ }^{13} \mathrm{CO}$ emission integrated over the velocity range $[0,12] \mathrm{km} \mathrm{s}^{-1}$. In the construction of this map, we have removed the contribution of the error beam to the observed intensities and expressed the resulting intensities on the corrected main beam scale, $T_{\mathrm{MB}, \mathrm{c}}$ (Bensch et al. 2001; Brunt et al., in prep.; Mottram $\&$ Brunt, in prep.) The map is 2048 pixels $\times 1529$ pixels across, corresponding to $28 \mathrm{pc} \times 21 \mathrm{pc}$ at a distance of $140 \mathrm{pc}$ (Elias 1978).

Following the procedure described in Sect. 2, we first estimate the variance in the normalized projected field. For this initial analysis, we use the entire field as represented in Fig. 1 with no thresholding of the intensities. We assume that the ${ }^{13} \mathrm{CO}$ integrated intensity, $I_{13}$, is linearly proportional to the column density, $N$. The advantages and disadvantages of this assumption are discussed below in Sect. 3.2, along with an alternative estimate of $b$ using extinction data to calculate the normalized column density variance. Taking $I_{13} \propto N$, we calculate $\sigma_{N / N_{0}}^{2}=\sigma_{I_{13} / I_{0,13}}^{2}$, where $I_{0,13}$ is the mean intensity. The observed variance in the field is the sum of the signal variance, $\sigma_{I_{13}}^{2}$, and the noise variance, $\sigma_{\text {noise }}^{2}$. We measure $\sigma_{I_{13}}^{2}+\sigma_{\text {noise }}^{2}=2.81$ and $\sigma_{\text {noise }}^{2}=0.42$, giving $\sigma_{I_{13}}^{2}=2.39$ (units are all $\left.\left(\mathrm{K} \mathrm{km} \mathrm{s}^{-1}\right)^{2}\right)$. With a measured $I_{0,13}=1.06 \mathrm{~K} \mathrm{~km} \mathrm{~s}^{-1}$, we then find $\sigma_{N / N_{0}}^{2}=2.25$.

The power spectrum of the integrated intensity field is now calculated. We use a square field of size 2048 pixels $\times$ 2048 pixels in which the map is embedded, and compute the power spectrum using a Fast Fourier Transform. Application of tapers to smoothly roll-off the field edges had an insignificant effect on the result (see Brunt \& Mac Low 2004). We calculate the noise floor using the power spectrum of an integrated map made over signal-free channels in the data, and this is subtracted from the power spectrum of the field. The central portion of the power spectrum is shown in Fig. 2 to demonstrate the level of anisotropy present. The black and white contours (as appropriate to the greyscale level) show levels of equal power, obtained from a smoothed version of the power spectrum for clarity, and the red circles are overlayed to show what would be expected for a fully isotropic power spectrum. While Taurus is often considered "elongated" or "filamentary", the power spectrum of ${ }^{13} \mathrm{CO}$ emission is in fact reasonably isotropic over most spatial frequencies. 


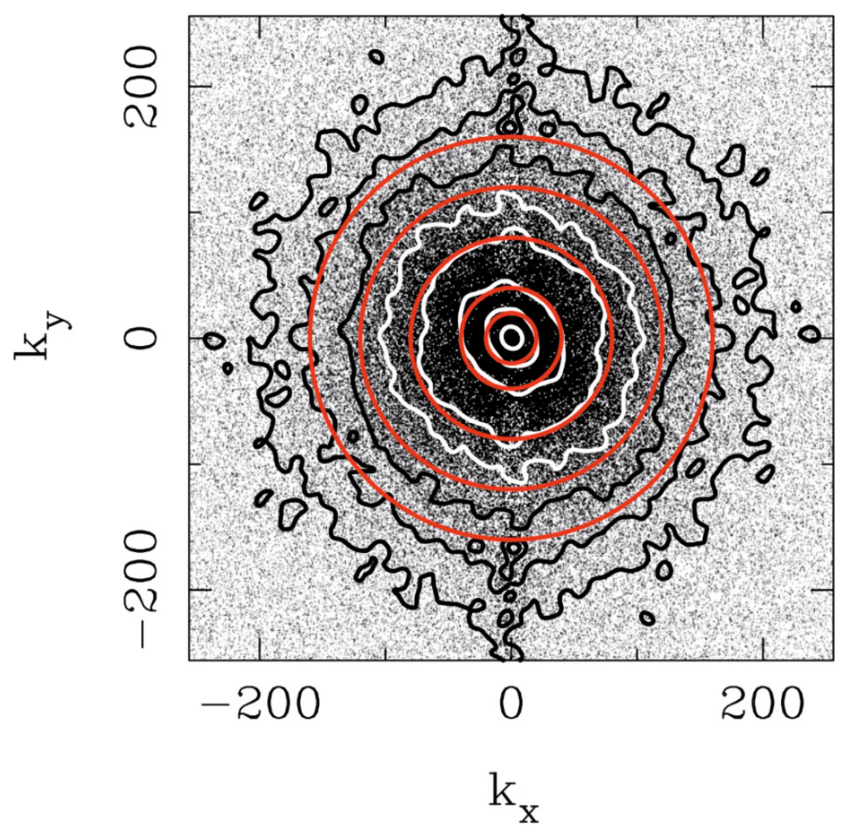

Fig. 2. Central portion of the power spectrum of the Taurus ${ }^{13} \mathrm{CO} J=$ 1-0 integrated intensity map. The black/white contours (smoothed for clarity) show levels of equal power, while the red circles represent an isotropic power spectrum for reference.

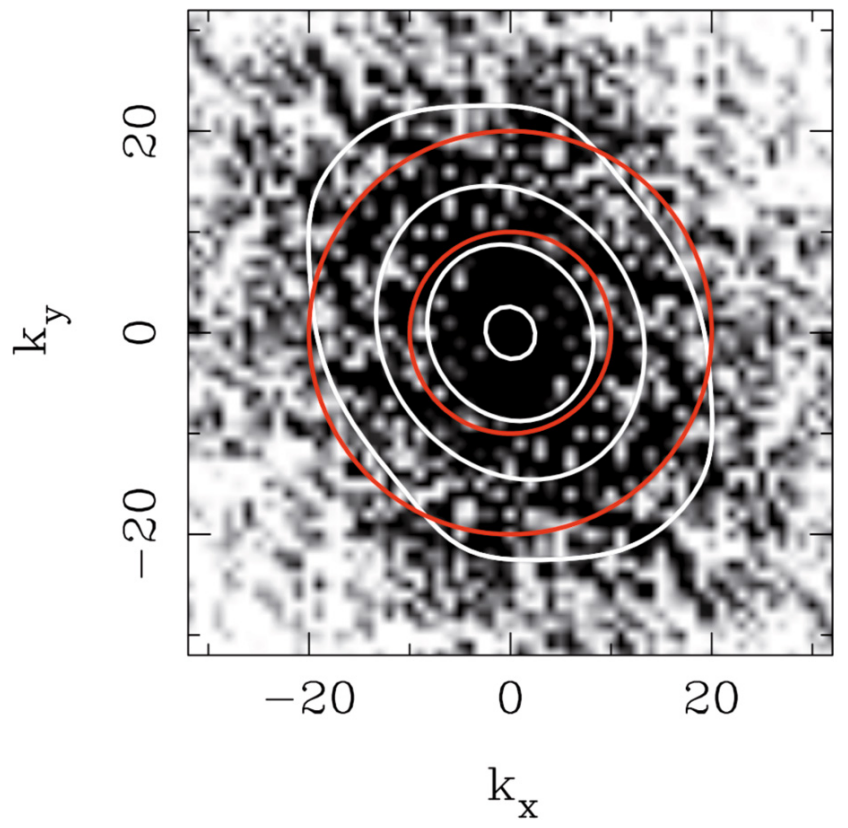

Fig. 3. Zoom in on the central portion of the power spectrum of Fig. 3. The white contours (smoothed for clarity) show levels of equal power, while the red circles represent an isotropic power spectrum for reference.

There is some evidence of anisotropy at the larger spatial scales (lower $k$ ), and we further demonstrate this in Fig. 3, which shows a zoom in on the smaller $k$ range of the power spectrum. Figure 3 shows that there is a preferred range in $k$ for anisotropy to be present, occurring at spatial frequencies near $k=20$.

Figure 4 shows the angular average of the power spectrum, $P_{N / N_{0}}(k)$, with the noise floor subtracted and the beam pattern divided out. The heavy lines in Fig. 4 display fitted power law forms $\left(P_{N / N_{0}}(k) \propto k^{-\alpha}\right)$ to two distinct $k$-ranges in the power

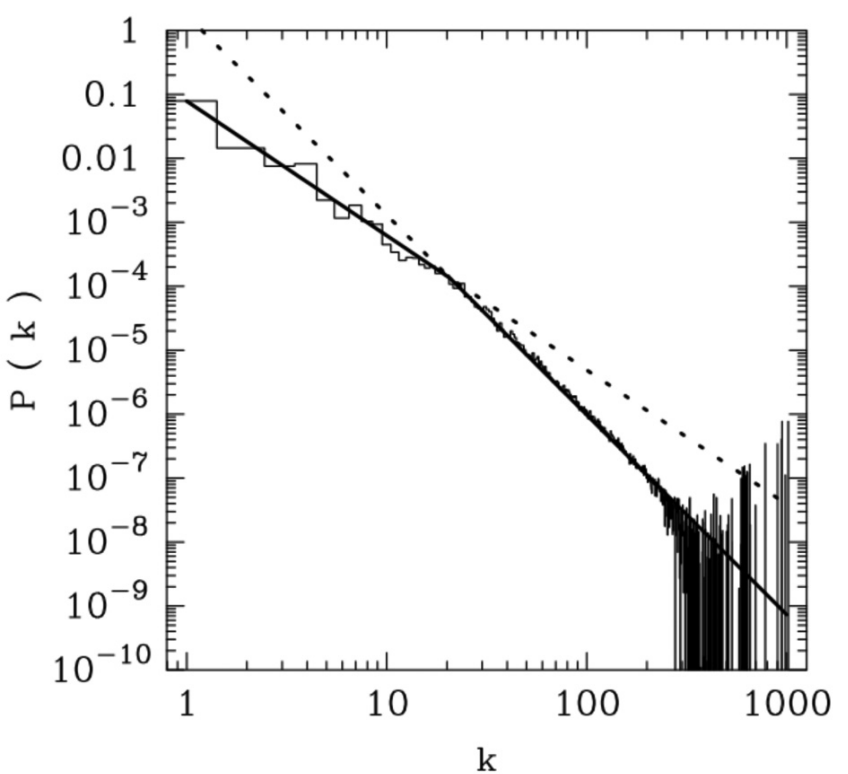

Fig. 4. Angular average of the ${ }^{13} \mathrm{CO} J=1-0$ integrated intensity power spectrum (histogram) corrected for the noise floor and beam pattern. The solid lines show the fitted power laws $P(k) \propto k^{-2.1}$ and $P(k) \propto k^{-3.1}$, for the ranges $k<21$ and $k>21$ respectively. The dashed lines show the continuation of the fitted power laws for reference.

spectrum. The dotted lines continue the fitted forms beyond their respective ranges for comparison. For $k<21$, the best fitting spectral slope is $\alpha=2.1$, while for $k>21$ we find $\alpha=3.1$. It is notable that the spectral break occurs at the preferred scale for anisotropy to be distinguishable in the power spectrum. The wavelength corresponding to a spatial frequency of $k=21$ is $\sim 0.5$ degrees, or $\sim 1.2$ pc. Previously, Blitz \& Williams (1997), using ${ }^{13} \mathrm{CO}$ data but not using the power spectrum, identified a characteristic scale of $\sim 0.25-0.5 \mathrm{pc}$ at which the structure of antenna temperature histograms changes notably. Hartmann (2002) also noted a characteristic separation scale of $\sim 0.25 \mathrm{pc}$ in the distribution of young low mass stars in Taurus. These scales are comparable to about $1 / 4$ the wavelength of the spectral break in the power spectrum. The orientation and scale of the anisotropy suggests that it arises from the repeated filament structure of Taurus, as suggested by Hartmann (2002).

Figures 2 and 3 show that the assumption of isotropy holds reasonably well in $2 \mathrm{D}$, but this is not necessarily a guarantee of isotropy in 3D, which, nevertheless, we must assume. The presence of a break in the power spectrum can be accommodated by Eq. (1) which makes no assumptions, other than isotropy, on the form of the power spectrum. Evaluating Eq. (1), we find that $R_{p}=0.029$, where the subscript $p$ reflects the fact that padding was employed in the power spectrum calculation. Evaluating Eq. (2) with $\sigma_{N / N_{0}}^{2}=2.25$ and $\eta=\left(\lambda_{p x} \lambda_{p y} / \lambda_{x} \lambda_{y}\right)^{\frac{1}{2}}=1.157$, we find that $\sigma_{\rho / \rho_{0}}^{2}=74.23$. The line-of-sight extent of the field assumed for this calculation is $\sqrt{28 \times 21} \mathrm{pc}=24.25 \mathrm{pc}$.

Given the high estimated sonic Mach number in Taurus (see below) it is unlikely that large scale strong anisotropies like those seen in sub-Alfvénic conditions will be present due to magnetic fields (BFP). Gravitational collapse along magnetic field lines could in principle generate anisotropy, and indeed the small anisotropy identified in the power spectrum is oriented as expected in this case (Heyer et al. 1987).

To estimate the rms Mach number in Taurus, we now conduct an analysis of the velocity field. Using the ${ }^{13} \mathrm{CO}$ spectral 


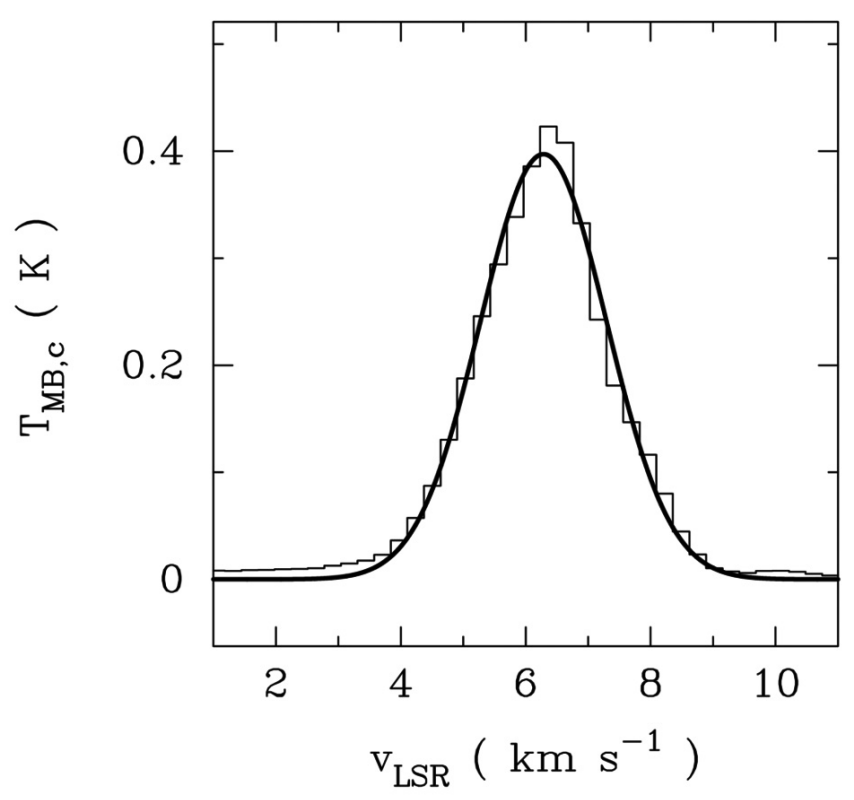

Fig. 5. Global mean spectral line profile for the Taurus ${ }^{13} \mathrm{CO}$ data (histogram). The heavy solid line is a Gaussian fit, with dispersion $\sigma_{v_{z}}^{2}=(1.02 \pm 0.06)\left(\mathrm{km} \mathrm{s}^{-1}\right)^{2}$.

line averaged over the field (Fig. 5), we measure the line-ofsight velocity dispersion, $\sigma_{v_{-}}^{2}=(1.02 \pm 0.06)\left(\mathrm{km} \mathrm{s}^{-1}\right)^{2}$ by fitting a Gaussian profile. The line-of-sight velocity dispersion includes contributions from all internal motions including those at the largest spatial scales arising from large scale turbulence (Ossenkopf \& Mac Low 2002; Brunt 2003; Brunt et al. 2009). Assuming isotropy, this implies a 3D velocity dispersion of $\sigma_{v}^{2}=3 \sigma_{v_{z}}^{2}=(3.06 \pm 0.18)\left(\mathrm{km} \mathrm{s}^{-1}\right)^{2}$. Taking the Taurus molecular gas to be at a temperature of $10 \mathrm{~K}$ (Goldsmith et al. 2008), with a mean molecular weight of 2.72 (Hildebrand 1983), the rms sonic Mach number is $M=17.6 \pm 1.8$, where the quoted error estimate is from spectral line fitting and uncertainties in the kinetic temperature (Goldsmith et al. 2008, report kinetic temperatures for the majority of Taurus between 6 and $12 \mathrm{~K}$; we have taken an uncertainty of $\pm 2 \mathrm{~K}$ ).

Combining these results, our observational estimate of $b$ therefore is $b=\sigma_{\rho / \rho_{0}} / M=0.49 \pm 0.06$, where we have applied an uncertainty of $10 \%$ to the estimated $\sigma_{\rho / \rho_{0}}^{2}$ (BFP). The uncertainty arising from the assumption of isotropy is difficult to quantify, and further progress on constraining $b$ will require the analysis of many more molecular clouds. Extension of this analysis to a larger sample of clouds will allow a test of whether $\sigma_{\rho / \rho_{0}}^{2}$ is indeed proportional to $M^{2}$ and may also allow investigation of whether $b$ changes due to varying degrees of compressive forcing of the turbulence (Federrath et al. 2008, 2009). The above estimate of the uncertainty in $b$ accounts for measurement errors on $M$ and known uncertainties in the calculation of $R$ from BFP. The true uncertainty is rather larger than this, as we discuss in the following sections.

\subsection{Measurement of $b$ using ${ }^{13} \mathrm{CO}$ and extinction data}

We now consider the consequences of the assumption of linear proportionality between the ${ }^{13} \mathrm{CO}$ integrated intensity and the column density. Goodman et al. (2009; see also Pineda et al. 2008) have recently evaluated different methods of measuring column densities in the Perseus molecular clouds. Their

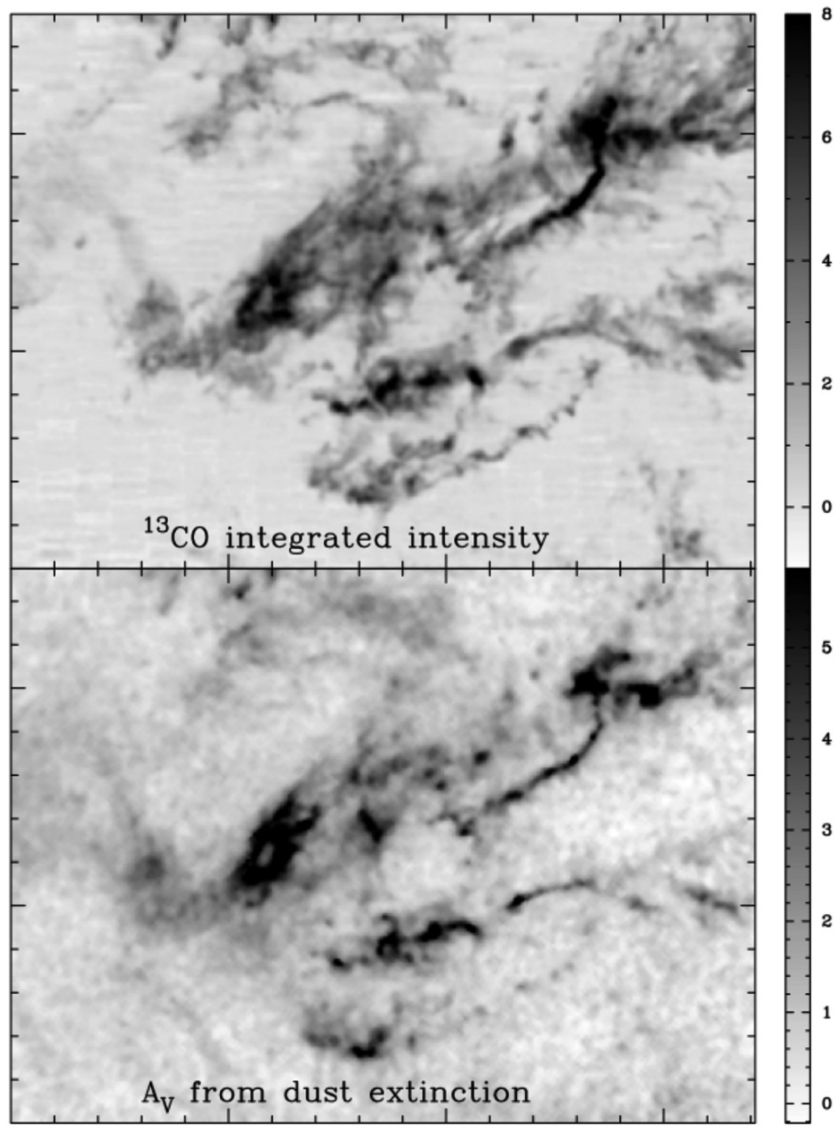

Fig. 6. Comparison of the ${ }^{13} \mathrm{CO}$ integrated intensity map with the dust extinction map of Froebrich et al. (2007).

findings may be simply summarised as follows: ${ }^{13} \mathrm{CO} J=1-0$ integrated intensity, $I_{13}$, is linearly proportional to $A_{V}$ estimated by dust extinction over a limited column density range (over about a decade or so in $I_{13}$ ), but is depressed by saturation and/or depletion in the high column density regime; in the low column density regime, $I_{13}$ is again depressed by lowered ${ }^{13} \mathrm{CO}$ abundance and/or subthermal excitation, and is insensitive to column densities below some threshold, as evidenced by an offset term in the $I_{13}-A_{V}$ relation. While all these factors impact on the point-to-point reliability of using $I_{13}$ to derive $N$, their effects on $\sigma_{I_{13} / I_{0,13}}^{2}$ and therefore on the estimated $\sigma_{N / N_{0}}^{2}$ are of more relevance here.

To empirically investigate the differences between using dust extinction and ${ }^{13} \mathrm{CO}$ integrated intensity as a measure of column density, we re-derived $\sigma_{N / N_{0}}^{2}$ using the dust extinction map generated from 2MASS data by Froebrich et al. (2007). In Fig. 6 we compare, on the same coordinate grid, the the dust extinction map with the ${ }^{13} \mathrm{CO}$ integrated intensity map convolved to the resolution of the extinction map ( 4 arcmin). The greyscale limits of each field were chosen to represent the fitted relation between the ${ }^{13} \mathrm{CO}$ integrated intensity, $I_{13}$, and the dust extinction, $A_{V}$, described below. While the overall spatial distributions of $I_{13}$ and $A_{V}$ are broadly similar, there are detailed differences in places, and column density traced by $A_{V}$ is present on the periphery of the cloud which is not traced by $I_{13}$.

The quantitative relation between $I_{13}$ and $A_{V}$ is shown in Fig. 7. To these data, we fitted a linear relationship of the form $A_{V}=A_{V, D}+C I_{13}$, where $A_{V, D}$ and $C$ are constants. We use three different regression methods (Isobe et al. 1990) and the fitted parameters, $A_{V, D}$ and $C$, are listed in Table 1, and the fitted lines 


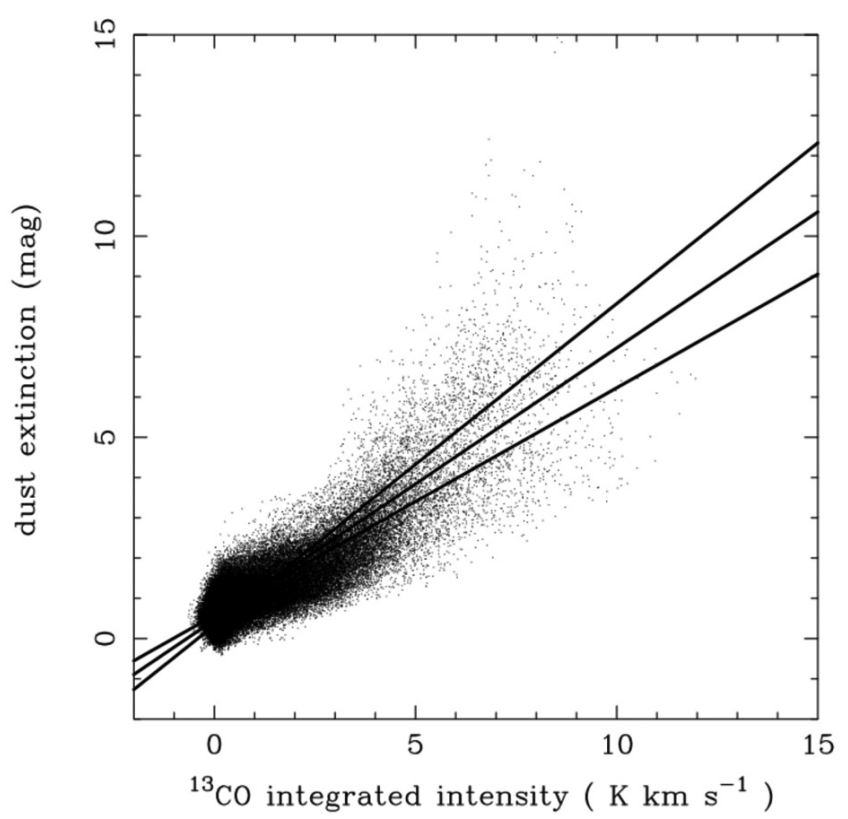

Fig. 7. Plot of dust extinction versus ${ }^{13} \mathrm{CO}$ integrated intensity, $I_{13}$, for the fields in Fig. 6. The solid lines are the fitted relations to $A_{V}=A_{V, D}+$ $C I_{13}$ listed in Table 1.

are overlayed in Fig. 7. While the scatter around these relations is quite large there is only a small number of positions in which significant saturation in $I_{13}$ is obvious.

Using the measured $A_{V}$ field to calculate $\sigma_{N / N_{0}}^{2}$, under the assumption that $A_{V}$ is proportional to column density, $N$, and accounting for a noise variance of 0.04 (Froebrich et al. 2007), we find that $\sigma_{N / N_{0}}^{2}=0.70$. This is significantly lower than $\sigma_{N / N_{0}}^{2}=2.25$ estimated through the ${ }^{13} \mathrm{CO}$ integrated intensities. There are two contributing factors to the lower value obtained using $A_{V}$. The first is simply that the fields shown in Fig. 6 are of lower angular resolution than the field shown in Fig. 1. A calculation of the normalized column density variance using the lowered resolution $I_{13}$ map results in $\sigma_{N / N_{0}}^{2}=1.98$, which is lower than that calculated from the higher resolution field, but still a factor of 2.83 greater than that calculated from the $A_{V}$ field at the same angular resolution. (Further discussion of the resolution dependence is given below and in Sect. 3.3.)

The second, and dominant, factor contributing to the lower $\sigma_{N / N_{0}}^{2}$ measured from the $A_{V}$ field is the presence of the offset term, $A_{V, D}$ in the $I_{13}-A_{V}$ relations given above. For reference, note that the addition of a constant positive offset to $N$ will raise $N_{0}$ but leave $\sigma_{N}^{2}$ unchanged, thereby lowering $\sigma_{N / N_{0}}^{2}$. The true situation is more complicated that this, since the low column density material is also structured (i.e. contributes variance) and it is possible that the column density variance scales with column density (Lada et al. 1994). It is questionable whether this material should be included in the column density budget. The excess column density (roughly quantified by $A_{V, D}$ ) is associated with atomic gas and possibly also diffuse molecular gas $\left(\mathrm{H}_{2}\right)$ with little or no ${ }^{13} \mathrm{CO}$.

Material in this regime does not contribute to the ${ }^{13} \mathrm{CO}$ emission, and therefore also not to the mean spectral line profile, from which the Mach number is estimated. If the material is predominantly atomic with a possible contribution from diffuse $\mathrm{H}_{2}$, it is likely to be physically warmer than the interior regions of the cloud traced by ${ }^{13} \mathrm{CO}$, thereby complicating the assumption of
Table 1. Fitted parameters to $A_{V}=A_{V, D}+C I_{13}$

\begin{tabular}{lcc}
\hline \hline Regression method & $A_{V, D}(\mathrm{mag})$ & $C\left(\mathrm{mag} /\left(\mathrm{K} \mathrm{km} \mathrm{s}^{-1}\right)\right)$ \\
\hline$A_{V}$ on $I_{13}$ & $0.579 \pm 0.002$ & $0.565 \pm 0.003$ \\
$I_{13}$ on $A_{V}$ & $0.331 \pm 0.004$ & $0.799 \pm 0.004$ \\
Bisector & $0.462 \pm 0.003$ & $0.676 \pm 0.003$ \\
\hline
\end{tabular}

isothermality employed in most of the numerical models and in our calculation of the Mach number. Similarly, ${ }^{13} \mathrm{CO}$ emission from the subthermally excited (but also likely physically warmer) envelope regions will contribute to both to $I_{13}$ and to the mean line profile, but at a reduced level in relation to its true column density. In addition, while the "molecular cloud" part of the extinction is confined to a small region of around $24 \mathrm{pc}$ extent at a distance of $140 \mathrm{pc}$, how the "diffuse" part of the extinction is generated is rather less well defined, and may include contributions from all regions along the line-of-sight to Taurus (and beyond). Consequently, the extinction zero level is quite uncertain. We argue therefore that the selection of material by ${ }^{13} \mathrm{CO}$ is in fact beneficial to our aims: it preferentially selects the interior regions of the molecular cloud, to which the assumption of isothermality is likely to apply, and it limits the extinction budget to a spatially-confined region at the distance of the cloud. Note also that material contributing to $I_{13}$ (and therefore $N$, by assumption) contributes in the same proportion to the mean line profile.

Inclusion of opacity corrections, using ${ }^{12} \mathrm{CO}$ data, also potentially cause as many problems as they solve. A spatially uniform opacity factor obviously has no impact on the calculated $\sigma_{N / N_{0}}^{2}$ due to the normalization. In principle, the compression of $I_{13}$ in the high column density regime can be alleviated, but this effect is likely to be small - particularly since the stronglysaturated column densities account for a small fraction of the data. Goodman et al. (2009) discuss numerous problems in the ${ }^{12} \mathrm{CO}$-based correction for opacity and excitation in the low column density regime; we also point out here that this is further complicated by the fact that ${ }^{12} \mathrm{CO}$ and ${ }^{13} \mathrm{CO}$ trace different material because of different levels of self-shielding and radiative trapping. If opacity corrections to $I_{13}$ are made, then for consistency, detailed (largely impractical) opacity corrections should be made to the line profiles from which the Mach number is calculated, although for this the point-of-diminishing-returns has certainly long since been reached.

To investigate the effect of the inclusion of the diffuse component, we calculate $\sigma_{N / N_{0}}^{2}$ from the field $A_{V}^{\prime}=A_{V}-A_{V, D}$. For the first of the fitted relations in Table $1\left(A_{V, D}=0.579\right)$ we find $\sigma_{N / N_{0}}^{2}=2.73$; from the second $\left(A_{V, D}=0.331\right)$ we find $\sigma_{N / N_{0}}^{2}=1.36$, and from the third, bisector method $\left(A_{V, D}=\right.$ 0.462 ) we find $\sigma_{N / N_{0}}^{2}=1.91$. These values may be summarized in a combined measurement, with associated uncertainties, of $\sigma_{N / N_{0}}^{2}=1.91_{-0.55}^{+0.82}$, which is more compatible with $\sigma_{N / N_{0}}^{2}=1.98$ calculated from the ${ }^{13} \mathrm{CO}$ field at the same angular resolution. This is not surprising, as $A_{V}^{\prime}$ is, to a good approximation, linearly correlated with $I_{13}$, and the value of the coefficient $C$ is irrelevant as we use normalized variances.

The dominant contribution to the uncertainty therefore comes from the choice made for the appropriate treatment of the diffuse component. We argue that the use of ${ }^{13} \mathrm{CO}$, or $A_{V}^{\prime}$, to calculate $\sigma_{N / N_{0}}^{2}$ is better-motivated, but we cannot resolve this issue further at present. 


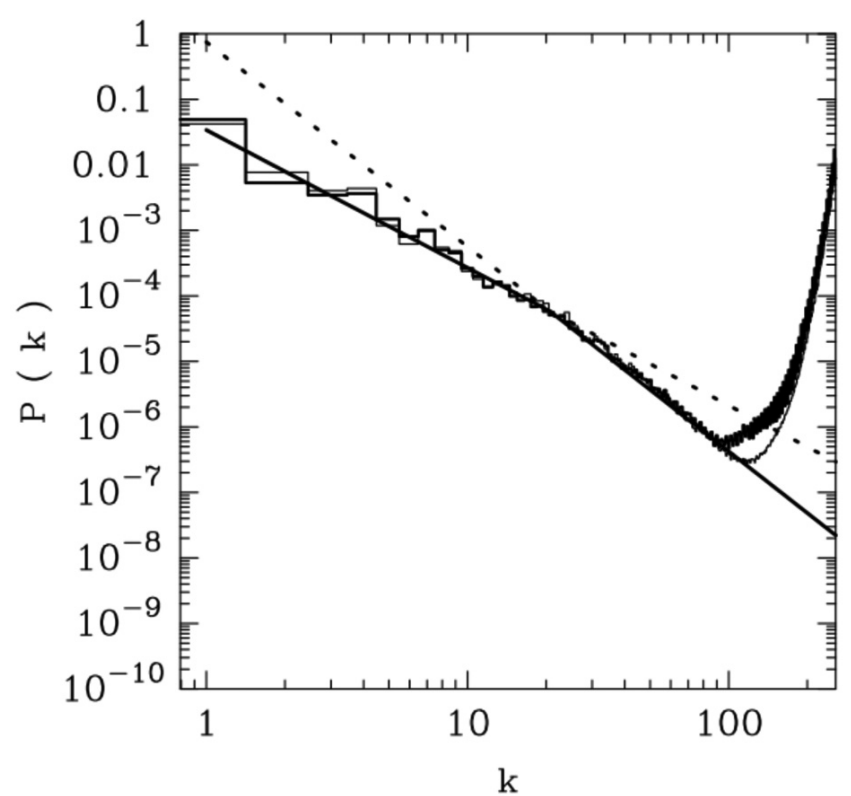

Fig. 8. Power spectra of the fields in Fig. 6. The light histogram is the power spectrum of ${ }^{13} \mathrm{CO}$ integrated intensity; the heavy histogram is the power spectrum of dust extinction. Both fields were scaled to the same global variance of unity before calculating the power spectra. The power spectra were corrected for the beam pattern but not the noise floor, resulting in an upturn at high wavenumber. The straight and dashed lines are the fits from Fig. 4.

We have noted above that the $\sigma_{N / N_{0}}^{2}$ calculated from ${ }^{13} \mathrm{CO}$ is, naturally, lower in the reduced resolution field. Ideally, one should use the highest resolution data available from which to calculate $\sigma_{N / N_{0}}^{2}$, and this raises the question of how the $A_{V^{-}}$ calculated value of $\sigma_{N / N_{0}}^{2}$ may behave at increased resolution. To investigate this, we calculate the power spectra of the ${ }^{13} \mathrm{CO}$ and $A_{V}$ fields of Fig. 6, after scaling each to the same global variance of unity (the mean of the field is irrelevant). The angular averages of the power spectra are shown in Fig. 8. In these spectra, we have applied "beam" corrections, but have not attempted removal of the noise floor.

It is remarkable that, despite detailed differences between the two maps, the power spectra are almost indistinguishable. The fitted power-laws from Fig. 4 are overlayed for reference - detailed correspondance between the two power spectra is notable all the way down to the noise floor(s) near $k \approx 100$. Comparison of the low resolution ${ }^{13} \mathrm{CO}$ power spectrum to the fitted slope at high wavenumber from Fig. 1 shows that the noise signature turns on very abruptly, and the power spectrum is very reliable up to this point - this is a useful benchmark to gauge the equivalence of the two low resolution spectra. Previously, Padoan et al. (2006) have reported differences between ${ }^{13} \mathrm{CO}$ and extinction power spectra in Taurus, which is not seen in our analysis. The origin of this discrepancy may lie in the different extinction maps used by Padoan et al. (those of Cambrésy 2002), but may also arise from the influence of the effective "beam" size that is not corrected for in their power spectra as the resolution is varied.

The effective equivalence of the ${ }^{13} \mathrm{CO}$ and $A_{V}$ power spectra support our suggestion above that it is in the approximately uniform "diffuse" component that the principal statistical differences (at least those relevant to our analysis) between ${ }^{13} \mathrm{CO}$ and $A_{V}$ are manifest. This, in turn, suggests that the $A_{V}$-derived value of $\sigma_{N / N_{0}}^{2}$ that would be obtained at the higher resolution afforded by the ${ }^{13} \mathrm{CO}$ data will increase in a similar manner to that seen in the ${ }^{13} \mathrm{CO}$ - i.e. an increase from 1.98 to 2.25 , or a factor of $\sim 1.14$. Applying this to the $A_{V}^{\prime}$-based measurements, our estimate for $\sigma_{N / N_{0}}^{2}$ at the highest resolution available is $\sigma_{N / N_{0}}^{2}=2.18_{-0.63}^{+0.93}$. Converting this to the $3 \mathrm{D}$ variance using Eq. (2) (with $\eta=1.157$ and $R_{p}=0.029$ ) we find that $\sigma_{\rho / \rho_{0}}^{2}=72.1_{-18.7}^{+27.8}$. Taking into account the measured Mach number, $M=17.6 \pm 1.8$, and applying an additional $10 \%$ uncertainty on the $3 \mathrm{D}$ variance arising from the calculation of $R$, we arrive at a measured $b=0.48_{-0.11}^{+0.15}$. (For reference, if $\sigma_{N / N_{0}}^{2}$ is calculated from $A_{V}$ without subtraction of the diffuse component, we find $b=0.32$.)

\subsection{The effects of unresolved variance}

As we have noted above, the measured variance of a field observed at finite resolution is necessarily a lower limit to the true variance of the field. An obvious question then arises: what about structure (variance) at scales below the best available resolution?

If we assume that the observed slope of the power spectrum at high wavenumbers continues to a maximum wavenumber, $k_{\mathrm{c}}$, beyond which no further structure is present, we can estimate the amount of variance not included in our calculation (see BFP). It has been suggested that $k_{\mathrm{c}}$ may be determined by the sonic scale: turbulent motions are supersonic above this scale and subsonic below it (Vázquez-Semadeni et al. 2003; Federrath et al. 2009). Taking $\sigma_{v} \propto L^{0.5}$ (Heyer \& Brunt 2004), we estimate the sonic scale in Taurus as $L_{\mathrm{s}}=L_{0} / M^{2} \approx 0.08 \mathrm{pc}$ where $L_{0} \approx 25 \mathrm{pc}$ is the linear size of the cloud from Fig. 1 , and $M=17.6$ is the Mach number from above. This is comparable to the spatial resolution of the data $(0.03 \mathrm{pc})$, which suggests that our measurement of $\sigma_{\rho / \rho_{0}}^{2}$ does not significantly underestimate the true value if structure is suppressed below the sonic scale. Taking $k_{\mathrm{c}} \longrightarrow \infty$ on the other hand would result in a factor of $\sim 2$ underestimation of $\sigma_{\rho / \rho_{0}}^{2}$ and therefore a factor of $\sim \sqrt{2}$ underestimation of $b$. Our value of $b$ must therefore be considered as a lower limit: the power spectrum (Fig. 4 ) is not well measured near the estimated sonic scale $\left(k_{\mathrm{s}} \sim 350\right)$, and further high resolution investigations are needed to resolve this issue.

\section{Summary}

We have provided an estimate of the parameter $b$ in the proposed $\sigma_{\rho / \rho_{0}}^{2}=b^{2} M^{2}$ relation for supersonic turbulence in isothermal gas, using ${ }^{13} \mathrm{CO}$ observations of the Taurus molecular cloud. Using ${ }^{13} \mathrm{CO}$ and $2 \mathrm{MASS}$ extinction data, we find $b=0.48_{-0.11}^{+0.15}$, which is consistent with the value originally proposed by Padoan et al. (1997a,b) and characteristic of turbulent forcing which includes a mixture of both solenoidal and compressive modes (Federrath et al. 2009). Our value of $b$ is a lower limit if significant structure exists below the resolution of our observations (effectively, below the sonic scale). If diffuse material is included in the column density budget then we find a somewhat lower value of $b=0.32$, although in this case there are some questions over the assumption of isothermality in the Mach number calculation. Our most conservative statement is therefore that $b$ is constrained to lie in the range $0.3 \lessgtr b \lessgtr 0.8$, which is comparable to the range of current numerical estimates. However, the $\sigma_{\rho / \rho_{0}}^{2}-M^{2}$ relation has been tested to only relatively low Mach numbers $(M \lesssim 7)$ in comparison to that in Taurus, so further numerical exploration of the high Mach number regime should be carried out. In principle, gravitational amplification of turbulently-generated density enhancements could 
increase $\sigma_{\rho / \rho_{0}}^{2}$ at roughly constant Mach number, with gravity possibly acting analogously to compressive forcing. Numerical investigation of the role of gravity in the $\sigma_{\rho / \rho_{0}}^{2}-M^{2}$ would be worthwhile.

Further analysis of a larger sample of molecular clouds is needed to investigate the linearity of the $\sigma_{\rho / \rho_{0}}^{2}-M^{2}$ relation and possible variations in $b$. As our main sources of uncertainty arise because of questions over how to treat the diffuse (likely atomic) regime and how to account for unresolved density variance, further progress on improving the accuracy of measuring $b$ ultimately must involve answering what is meant by "a cloud" (Ballesteros-Paredes et al. 1999) and down to what spatial scale is significant structure likely to be present?

Acknowledgements. I would like to thank Dan Price and Christoph Federrath for excellent encouragement, advice, and scientific insight. Figure A.1 was kindly provided by Dan Price and Matthew Bate. This work was supported by STFC Grant ST/F003277/1 to the University of Exeter, Marie Curie Re-Integration Grant MIRG-46555, and NSF grant AST 0838222 to the Five College Radio Astronomy Observatory. C.B. is supported by an RCUK fellowship at the University of Exeter, UK. The Five College Radio Astronomy Observatory was supported by NSF grant AST 0838222 . This publication makes use of data products from 2MASS, which is a joint project of the University of Massachusetts and the Infrared Processing and Analysis Center/California Institute of Technology, funded by the National Aeronautics and Space Administration and the National Science

\section{Appendix A: Zero padding}

Brunt et al. (2009) considered an observable 2D field, $F_{2}$, which is produced by averaging a $3 \mathrm{D}$ field, $F_{3}$, over the line-of-sight axis. The field $F_{2}$ was considered to be distributed in a square region with a scale ratio (number of pixels along each axis) of $\lambda$. Assuming isotropy, the 3D field then is distributed in a cubical region, again of scale ratio $\lambda$.

A column density field, $N$, is produced by integrating the density field, $\rho$, along the line-of-sight, rather than averaging. To conform to the conditions set out by BFP, it is necessary to work with the normalised column density field and density field, which are obtained by dividing each field by their respective mean values, $N_{0}$ and $\rho_{0}$. The variances calculated from these fields are $\sigma_{N / N_{0}}^{2}$ and $\sigma_{\rho / \rho_{0}}^{2}$ respectively. The latter is the quantity required to test the theoretical prediction: $\sigma_{\rho / \rho_{0}}^{2}=b^{2} M^{2}$.

Consider a $2 \mathrm{D}$ field, $F_{2}$, with mean value $F_{2,0}$, which is the projection of a 3D field, $F_{3}$, with mean $F_{3,0}$. The normalised variance, $\sigma_{2}^{2}=\sigma_{F_{2} / F_{2,0}}^{2}$, can be calculated for a square image, of scale ratio $\lambda$, via:

$\sigma_{2}^{2}=\frac{\left\langle F_{2}^{2}\right\rangle-F_{2,0}^{2}}{F_{2,0}^{2}}=\frac{\left\langle F_{2}^{2}\right\rangle}{F_{2,0}^{2}}-1$

where:

$\left\langle F_{2}^{2}\right\rangle=\frac{\sum_{i=1}^{\lambda} \sum_{j=1}^{\lambda} F_{2}^{2}(i, j)}{\lambda^{2}}$

$F_{2,0}=\left\langle F_{2}\right\rangle=\frac{\sum_{i=1}^{\lambda} \sum_{j=1}^{\lambda} F_{2}(i, j)}{\lambda^{2}}$

and $F_{2}(i, j)$ is the value of $F_{2}$ at the pixel $(i, j)$.

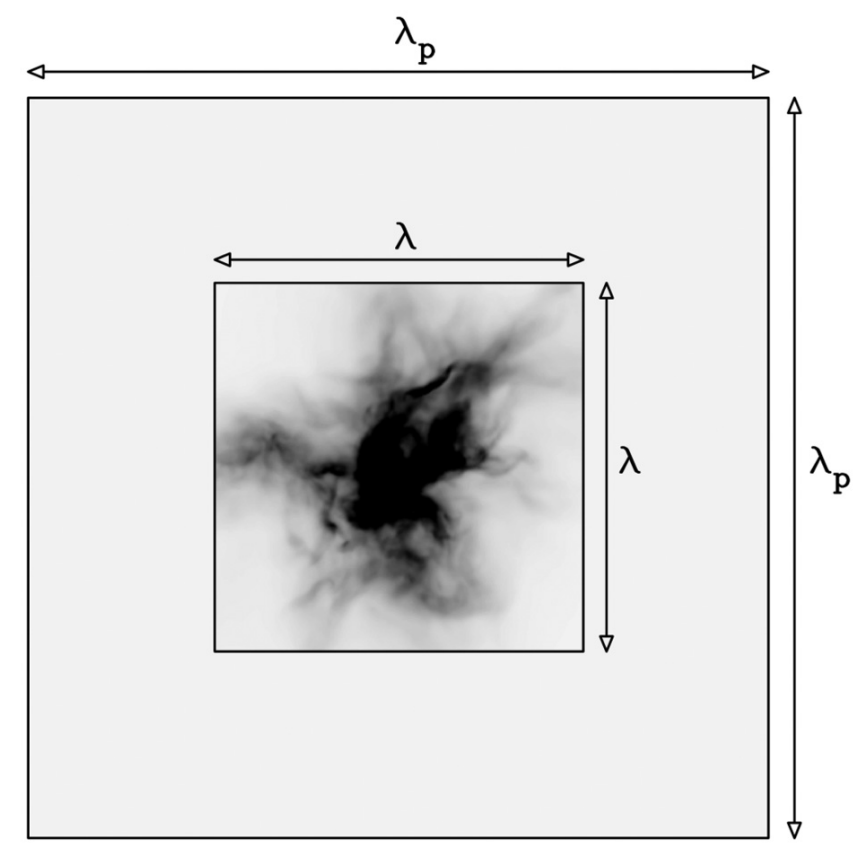

Fig. A.1. Schematic illustration of zero-padding.

Similarly, the normalised variance of $F_{3}$ is $\sigma_{3}^{2}=\sigma_{F_{3} / F_{3,0}}^{2}$, given by:

$\sigma_{3}^{2}=\frac{\left\langle F_{3}^{2}\right\rangle-F_{3,0}^{2}}{F_{3,0}^{2}}=\frac{\left\langle F_{3}^{2}\right\rangle}{F_{3,0}^{2}}-1$

where:

$\left\langle F_{3}^{2}\right\rangle=\frac{\sum_{i=1}^{\lambda} \sum_{j=1}^{\lambda} \sum_{k=1}^{\lambda} F_{3}^{2}(i, j, k)}{\lambda^{3}}$
$F_{3,0}=\left\langle F_{3}\right\rangle=\frac{\sum_{i=1}^{\lambda} \sum_{j=1}^{\lambda} \sum_{k=1}^{\lambda} F_{3}(i, j, k)}{\lambda^{3}}$

and $F_{3}(i, j, k)$ is the value of $F_{3}$ at the pixel $(i, j, k)$.

If $F_{2}$ is now zero-padded to a scale ratio of $\lambda_{p}$ (shown schematically in Fig. A.1), the normalised variance, $\sigma_{2 p}^{2}$, of the resulting field, $F_{2 p}$, is given by:

$\sigma_{2 p}^{2}=\frac{\left\langle F_{2 p}^{2}\right\rangle-F_{2 p, 0}^{2}}{F_{2 p, 0}^{2}}=\frac{\left\langle F_{2 p}^{2}\right\rangle}{F_{2 p, 0}^{2}}-1$

where now:

$\left\langle F_{2 p}^{2}\right\rangle=\frac{\sum_{i=1}^{\lambda_{p}} \sum_{j=1}^{\lambda_{p}} F_{2 p}^{2}(i, j)}{\lambda_{p}^{2}}=\left\langle F_{2}^{2}\right\rangle \frac{\lambda^{2}}{\lambda_{p}^{2}}=\left\langle F_{2}^{2}\right\rangle / \eta^{2}$

$F_{2 p, 0}=\left\langle F_{2 p}\right\rangle=\frac{\sum_{i=1}^{\lambda_{p}} \sum_{j=1}^{\lambda_{p}} F_{2 p}(i, j)}{\lambda_{p}^{2}}=\left\langle F_{2}\right\rangle \frac{\lambda^{2}}{\lambda_{p}^{2}}=\left\langle F_{2}\right\rangle / \eta^{2}$ 
where we have defined $\eta=\lambda_{p} / \lambda$, and noted that:

$$
\begin{aligned}
& \sum_{i=1}^{\lambda_{p}} \sum_{j=1}^{\lambda_{p}} F_{2 p}(i, j)=\sum_{i=1}^{\lambda} \sum_{j=1}^{\lambda} F_{2}(i, j) \\
& \sum_{i=1}^{\lambda_{p}} \sum_{j=1}^{\lambda_{p}} F_{2 p}^{2}(i, j)=\sum_{i=1}^{\lambda} \sum_{j=1}^{\lambda} F_{2}^{2}(i, j)
\end{aligned}
$$

since $F_{2 p}$ is zero outside the region where $F_{2}$ is defined.

Similarly:

$$
\begin{aligned}
\sigma_{3 p}^{2} & =\frac{\left\langle F_{3 p}^{2}\right\rangle-F_{3 p, 0}^{2}}{F_{3 p, 0}^{2}}=\frac{\left\langle F_{3 p}^{2}\right\rangle}{F_{3 p, 0}^{2}}-1 \\
\left\langle F_{3 p}^{2}\right\rangle & =\frac{\sum_{i=1}^{\lambda_{p}} \sum_{j=1}^{\lambda_{p}} \sum_{k=1}^{\lambda_{p}} F_{3 p}^{2}(i, j, k)}{\lambda_{p}^{2}}=\left\langle F_{3}^{2}\right\rangle \frac{\lambda^{3}}{\lambda_{p}^{3}}=\left\langle F_{3}^{2}\right\rangle / \eta^{3}
\end{aligned}
$$

$F_{3 p, 0}=\left\langle F_{3 p}\right\rangle=\frac{\sum_{i=1}^{\lambda_{p}} \sum_{j=1}^{\lambda_{p}} \sum_{k=1}^{\lambda_{p}} F_{3 p}(i, j)}{\lambda_{p}^{2}}=\left\langle F_{3}\right\rangle \frac{\lambda^{3}}{\lambda_{p}^{3}}=\left\langle F_{3}\right\rangle / \eta^{3}$.

Combining these results, we find:

$$
\begin{aligned}
& \sigma_{2 p}^{2}=\left(\sigma_{2}^{2}+1\right) \eta^{2}-1 \\
& \sigma_{3 p}^{2}=\left(\sigma_{3}^{2}+1\right) \eta^{3}-1 .
\end{aligned}
$$

Application of the BFP method to the zero-padded field yields the ratio of $2 \mathrm{D}$-to-3D normalised variance:

$R_{p}=\sigma_{2 p}^{2} / \sigma_{3 p}^{2}$

where $R_{p}$ is calculated using the power spectrum of the zeropadded field, and we have identified this by the subscript $p$ on $R$. The quantity of interest, however, is $\sigma_{3}^{2}$ which can be derived from the measured $\sigma_{2}^{2}$ and $R_{p}$ via:

$\sigma_{3}^{2}=\frac{1}{\eta^{3}}\left(1+\left(\left(\sigma_{2}^{2}+1\right) \eta^{2}-1\right) / R_{p}\right)-1$,

obtained through combining Eqs. (A.15)-(A.17).

The BFP method assumes that the input image from which the $2 \mathrm{D}$ power spectrum is calculated is square. In the case that the observed field is not square, but has dimensions $\lambda_{x} \times \lambda_{y}$, zero-padding to a square field of size $\lambda_{p x} \times \lambda_{p y}$ is required. If now $\eta$ is defined as:

$\eta=\sqrt{\lambda_{p x} \lambda_{p y} / \lambda_{x} \lambda_{y}}$

this allows $\sigma_{3}^{2}$ to be calculated via Eq. (A.18) under the assumption that the line-of-sight extent of the field $F_{3}$ is:

$\lambda_{z}=\sqrt{\lambda_{x} \lambda_{y}}$

Obviously, it is desirable that $\lambda_{x} \approx \lambda_{y}$ for the assumption of isotropy to be best satisfied.

\section{References}

Ballesteros-Paredes, J., Vázquez-Semadeni, E., \& Scalo, J. 1999, ApJ, 515, 286 Bensch, F., Stutzki, J., \& Heithausen, A. 2001, A\&A, 365, 285

Blitz, L., \& Willams, J. P. 1997, ApJ, 488, L145

Brunt, C. M. 2003, ApJ, 583, 280

Brunt, C. M., \& Mac Low, M.-M. 2004, ApJ, 604, 196

Brunt, C. M., Heyer, M. H., \& Mac Low, M.-M. 2009, A\&A, 504, 883

Brunt, C. M., Federrath, C., \& Price, D. J. 2010, MNRAS, 403, 1507 (BFP)

Elias, J. H. 1978, ApJ, 224, 857

Elmegreen, B. G. 2008, ApJ, 672, 1006

Federrath, C., Klessen, R. S., \& Schmidt, W. 2008, ApJ, 688, 79

Federrath, C., Roman-Duval, J., Klessen, R., Schmidt, W., \& Mac Low, M.-M. 2010, A\&A, 512, A81

Froebrich, D., Murphy, G. C., Smith, M. D., Walsh, J., \& del Burgo, C. 2007, MNRAS, 378, 1447

Goldsmith, P. F., Heyer, M. H., Narayanan, G., et al. 2008, ApJ, 680, 428 Goodman, A. A., Pineda, J. E., \& Schnee, S. L. 2009, ApJ, 692, 91 Hartmann, L. 2002, ApJ, 578, 914

Hennebelle, P., \& Chabrier, G. 2008, ApJ, 684, 395 Hennebelle, P., \& Chabrier, G. 2009, ApJ, 702, 1428

Heyer, M. H., \& Brunt, C. M. 2004, ApJ, 615, L45

Heyer, M. H., Vrba, F. J., Snell, R. L., et al. 1987, ApJ, 321, 855

Heyer, M. H., Gong, H., Ostriker, E., \& Brunt, C. 2008, ApJ, 680, 420

Hildebrand, R. H. 1983, QJRAS, 24, 267

Isobe, T., Feigelson, E. D., Akritas, M. G., \& Babu, G. J. 1990, ApJ, 364, 104

Kritsuk, A. G., Norman, M. L., Padoan, P., \& Wagner, R. 2007, ApJ, 665, 416

Krumholz, M. R., \& McKee, C. F. 2005, ApJ, 630, 250

Lada, C. J., Lada, E. A., Clemens, D. P., \& Bally, J. 1994, ApJ, 429, 694

Larson, R. B. 1981, MNRAS, 194, 809

Lemaster, M. N., \& Stone, J. M. 2008, ApJ, 682, L97

Mac Low, M.-M. 1999, ApJ, 524, 169

Narayanan, G., Heyer, M. H., Brunt, C. M., et al. 2008, ApJS, 177, 341

Ossenkopf, V., \& Mac Low, M.-M. 2002, A\&A, 390, 307

Padoan, P., \& Nordlund, A. 2002, ApJ, 576, 870

Padoan, P., \& Nordlund, Å. 2009, ApJ, submitted [arXiv: 0907 . 0248]

Padoan, P., Jones, B. J. T., \& Nordlund, Å. 1997a, ApJ, 474, 730

Padoan, P., Nordlund, A., \& Jones, B. J. T. 1997b, MNRAS, 288, 145

Padoan, P., Cambrésy, L., Juvela, M., et al. 2006, ApJ, 649, 807

Passot, T., \& Vázquez-Semadeni, E. 1998, PhRvE, 58, 4501

Pineda, J., Caselli, P., \& Goodman, A. A. 2008, ApJ, 679, 481

Vázquez-Semadeni, E. 1994, ApJ, 423, 681

Vázquez-Semadeni, E., Ballesteros-Paredes, J., \& Klessen, R. S. 2003, ApJ, 585, L131 